\title{
Fission Yield Calculation Method and its Effect in Nuclear Fuel Cell Homogenization Calculation
}

\author{
Zaki Su'ud ${ }^{\text {I) }}$, M. Ali Shafii ${ }^{\text {I) }}$, and Rida SNM(2) \\ 1) Nuclear and Biophysics Research Division, \\ Faculy of Mathematics and Natural Sciences, \\ Bandung Institute of Technology, \\ Jl. Ganesha 10, Bandung, Indonesia \\ ${ }^{2)}$ Doctoral student, Dept. Nuclear Engineering, Kyushu University, Japan \\ e-mail:szaki@fi.itb.ac.id
}

\begin{abstract}
Zero burn-up core capability can eliminate possible super prompt critical accident and make possible of inherent safety feature based on reactivity feedback mechanism. In this concept the maximum excess reactivity is limited below 1 \$ of reactivity so that possibility of super prompt accident such as in Chernobyl accident case can be eleminated. This is however need high quality of system analysis, and in this study the effect of fission yield calculation method on the nuclear fuel cell homogenization process is investigated and discussed. This study use SRAC code system to investigate the efect of reactor dependent fission yield distribution calculation. Calculation restults show that this process will has important improvement effect for long life high burnup core.
\end{abstract}

Keywords : Zero burn-up, Inherent safety, Fission yield, Neutron energy spectrum, Nuclear fuel cell homogenization

\section{Introduction}

After chernobyl accident there are a strong shift in the nuclear power plant safety paradigm which shifts toward inherent/safety paradigm. $\mathrm{Pb} / \mathrm{Pb}-\mathrm{Bi}$ cooled fast reactors are among new emerging next generation nuclear power plant with many advantages such as inherent safet capability against unprotected rod run out transient over power (UTOP) accident as well as unprotected loss of flow (ULOF) accident; ability to breed uranium 238 to be utilized efficiently, ability to burn nuclear waste, and economically competitive ${ }^{1-8)}$.

One of important feature of lead/lead-bismuth cooled fast reactors is the zero burn-up core capability which can eliminate possible super prompt critical accident and make possible of inherent safety feature based on reactivity feedback mechanism. In this concept the maximum excess reactivity is limited below 1 \$ of reactivity so that possibility of super prompt accident such as in Chernobyl accident case can be eleminated. This is however need high quality of system analysis as well as nuclear and material data to reduce calculation error so that its influence to the key design and safety parameters can be negligible. In this study the effect of fission yield calculation method on the nuclear fuel cell homogenization process is investigated and discussed. This development is important to support implementation process of zero burn-up concept in inherently safe fast reactors.

\section{Mathematical Model and Calculational Method}

In most of the nuclear fuel cell homogenization codes the fission yield distribution is calculated for several standard energy spectrum and during cell calculation process the user should select one of the nearest spectrum. In this study the fission yield calculation is recalculated according to the real reactor energy spectrum and the restults are compared to the previous common model.Detail mathematical model of the calculations can be divided into two parts as follows ${ }^{9-12)}$.

\subsection{Steady State Multigroup Diffusion Calculation}

Mathematical formulation of steady state multi group diffusion calculation can be written as follows

$$
\begin{aligned}
& -\vec{\nabla} \bullet D_{g} \vec{\nabla} \Psi_{g}(\vec{r}, t)+\sum_{r g} \Psi_{g}(\vec{r}, t)= \\
& \frac{x_{g}}{k_{e f f}} \sum_{g^{\prime}=1}^{G} \sum_{f g^{\prime}} \Psi_{g^{\prime}}(\vec{r}, t)+\sum_{g^{\prime}=1}^{G} \sum_{s g^{\prime} \rightarrow g} \Psi_{g^{\prime}}(\vec{r}, t)
\end{aligned}
$$

Where:

$g$ : energy group

$D$ : diffusion constant

$\Sigma_{r}$ : macroscopic cross section of removal

$\Sigma_{s}$ : macroscopic cross section of scattering

$\Sigma_{f}$ : macroscopic cross section of fission

$v$ : average neutron number produced in fission

$\phi$ : neutron flux

$\chi_{g}$ : fission spectrum of energy group g

$k_{\text {eff }}$ : Effective multiplication factor

\subsection{Effective FP yield data calculation}

The effective FP yield data can be calculated using linear interpolation of energy dependency as follows:

$$
y_{i, g}^{n, m}=\frac{\left(u_{g, a v}-u_{\text {high }}\right) y_{i, g, h i g h}^{n, m}+\left(u_{\text {low }}-u_{g, a v}\right) y_{i, g, \text { low }}^{n, m}}{u_{\text {low }}-u_{\text {high }}}
$$

where $y_{i, g}{ }^{n, m}$ independent fission yield of nuclide $\mathrm{n}$ for energy group g from fissile nuclide $m$ 
$y_{i, g, h i g h}{ }^{n, m}$ independent fission yield for nuclide $\mathrm{n}$ from fissile nuclide $\mathrm{m}$ at nearest upper energy of energy group $g$ (from ENDF/B or JENDL library);

$y_{i, g, l o w}{ }^{n, m}$ independent fission yield for nuclide $\mathrm{n}$ from fissile nuclide $\mathrm{m}$ at nearest lower energy of energy group $g$ (from ENDF/B or JENDL library)

$u_{g, a v}$ : average lethargy for energy group g

$u_{g, h i g h}$ : lethargy of nearest higher energy of energy group $g$

$u_{g, \text { low }}$ : lethargy of nearest lower energy of energy group $g$

Similarly, for cumulative fission yield we have

$$
y_{c, g}^{n, m}=\frac{\left(u_{g, a v}-u_{h i g h}\right) y_{c, g, h i g h}^{n, m}+\left(u_{\text {low }}-u_{g, a v}\right) y_{c, g, \text { low }}^{n, m}}{u_{\text {low }}-u_{\text {high }}}
$$

where :

$y_{c, g}{ }^{n}$ : cumulative fission yield of nuclide $\mathrm{n}$ for energy group g

$y_{c, g, h i g h}{ }^{n}$ : cumulative fission yield for nuclide $\mathrm{n}$ at nearest upper energy of energy group $g$ (from ENDF/B or JENDL library)

$y_{c, \text { g,low }}{ }^{n}$ : cumulative fission yield for nuclide $\mathrm{n}$ at nearest lower energy of energy group $g$ (from ENDF/B or JENDL library)

The total FP yield data for nuclide $\mathrm{n}$ is given as

$$
y_{i}^{n}=\frac{\sum_{g=1}^{G} \sum_{m} y_{i, g}^{n, m} N_{m} \sigma_{f, g, m} \phi_{g}}{\sum_{g=1}^{G} \sum_{m} N_{m} \sigma_{f, g, m} \phi_{g}}
$$

and

$$
y_{c}^{n}=\frac{\sum_{g=1}^{G} \sum_{m} y_{c, g}^{n, m} N_{m} \sigma_{f, g, m} \phi_{g}}{\sum_{g=1}^{G} \sum_{m} N_{m} \sigma_{f, g, m} \phi_{g}}
$$

where

$N_{m}$ : atomic density of fissile nuclide $\mathrm{m}$

$\sigma_{f, g, m}$ : microscopic fission cross section of nuclide $\mathrm{m}$ for energy $g$

$\varphi_{g}:$ neutron flux at energy g

\section{Calculation Results and Discussion}

In this study $\mathrm{Pb}-\mathrm{Bi}$ cooled RBEC fast reactor is taken as a calculation model. We then investigated the contribution of each fissile and fissionable material to the total fission but we got that the Pu-239 was dominant so we focus to process the fission yield data based on Pu-239 fission. Next we calculate the fission yield produced by fission in each energy group for each important nuclide. The example of the results for Ru-101 is given in Table 1.

Then we generated the total (summed over energy groups) fission yield data for each important nuclide ${ }^{12)}$ which results are shown in Table 2.

\begin{tabular}{|c|c|c|}
\hline $\begin{array}{l}\text { Energy } \\
\text { Group }\end{array}$ & Independent & Cumulative \\
\hline 1 & $1.349347 \mathrm{E}-08$ & $6.282428 \mathrm{E}-02$ \\
\hline 2 & $1.569870 \mathrm{E}-08$ & 6.142716E-02 \\
\hline 3 & $1.790394 \mathrm{E}-08$ & $6.003004 \mathrm{E}-02$ \\
\hline 4 & 2.010917E-08 & 5.863292E-02 \\
\hline 5 & $2.231440 \mathrm{E}-08$ & 5.723580E-02 \\
\hline 6 & 2.451964E-08 & 5.583868E-02 \\
\hline 7 & 2.672487E-08 & 5.444156E-02 \\
\hline 8 & $2.893010 \mathrm{E}-08$ & 5.304445E-02 \\
\hline 9 & 3.113534E-08 & 5.164733E-02 \\
\hline 10 & 1.328823E-08 & 5.900539E-02 \\
\hline 11 & $1.323276 \mathrm{E}-08$ & 5.909721E-02 \\
\hline 12 & 1.317729E-08 & 5.918903E-02 \\
\hline 13 & 1.312181E-08 & 5.928085E-02 \\
\hline 14 & 1.306634E-08 & 5.937266E-02 \\
\hline 15 & 1.301087E-08 & 5.946448E-02 \\
\hline 16 & 1.295539E-08 & 5.955630E-02 \\
\hline 17 & 1.289992E-08 & 5.964812E-02 \\
\hline 18 & $1.284445 \mathrm{E}-08$ & 5.973993E-02 \\
\hline 19 & 1.278897E-08 & 5.983175E-02 \\
\hline 20 & $1.273350 \mathrm{E}-08$ & 5.992356E-02 \\
\hline 21 & 1.267803E-08 & 6.001538E-02 \\
\hline 22 & 1.262255E-08 & 6.010720E-02 \\
\hline 23 & $1.256708 \mathrm{E}-08$ & 6.019901E-02 \\
\hline 24 & 1.251161E-08 & 6.029083E-02 \\
\hline 25 & 1.245613E-08 & 6.038265E-02 \\
\hline 26 & $1.240066 \mathrm{E}-08$ & 6.047447E-02 \\
\hline 27 & $1.234519 \mathrm{E}-08$ & $6.056628 \mathrm{E}-02$ \\
\hline 28 & 1.228971E-08 & 6.065810E-02 \\
\hline 29 & $1.223424 \mathrm{E}-08$ & 6.074992E-02 \\
\hline 30 & 1.217877E-08 & 6.084174E-02 \\
\hline 31 & $1.212329 \mathrm{E}-08$ & 6.093355E-02 \\
\hline 32 & 1.206782E-08 & 6.102537E-02 \\
\hline 33 & 1.201235E-08 & 6.111719E-02 \\
\hline 34 & 1.195687E-08 & 6.120900E-02 \\
\hline 35 & $1.190140 \mathrm{E}-08$ & 6.130082E-02 \\
\hline 36 & 1.184593E-08 & 6.139264E-02 \\
\hline 37 & 1.179046E-08 & 6.148445E-02 \\
\hline 38 & 1.173498E-08 & 6.157627E-02 \\
\hline 39 & 1.167951E-08 & 6.166809E-02 \\
\hline 40 & $1.162404 \mathrm{E}-08$ & $6.175990 \mathrm{E}-02$ \\
\hline 41 & $1.156856 \mathrm{E}-08$ & 6.185172E-02 \\
\hline 42 & 1.151309E-08 & 6.194354E-02 \\
\hline 43 & $1.145762 \mathrm{E}-08$ & $6.203536 \mathrm{E}-02$ \\
\hline 44 & 1.140214E-08 & 6.212717E-02 \\
\hline 45 & 1.134667E-08 & 6.221899E-02 \\
\hline 46 & $1.129120 \mathrm{E}-08$ & $6.231081 \mathrm{E}-02$ \\
\hline 47 & 1.123572E-08 & 6.240262E-02 \\
\hline 48 & 1.118025E-08 & 6.249444E-02 \\
\hline 49 & 1.112478E-08 & 6.258626E-02 \\
\hline 50 & $1.106930 \mathrm{E}-08$ & $6.267808 \mathrm{E}-02$ \\
\hline 51 & 1.101383E-08 & 6.276989E-02 \\
\hline 52 & $1.095836 \mathrm{E}-08$ & $6.286171 \mathrm{E}-02$ \\
\hline 53 & $1.090288 \mathrm{E}-08$ & 6.295352E-02 \\
\hline 54 & $1.084741 \mathrm{E}-08$ & 6.304534E-02 \\
\hline 55 & 1.079194E-08 & 6.313716E-02 \\
\hline 56 & 1.073647E-08 & 6.322897E-02 \\
\hline 57 & $1.068099 \mathrm{E}-08$ & $6.332079 \mathrm{E}-02$ \\
\hline 58 & 1.062552E-08 & 6.341261E-02 \\
\hline 59 & $1.057005 \mathrm{E}-08$ & 6.350443E-02 \\
\hline 60 & $1.051457 \mathrm{E}-08$ & 6.359624E-02 \\
\hline 61 & 1.045910E-08 & 6.368806E-02 \\
\hline 62 & 1.040363E-08 & 6.377988E-02 \\
\hline 63 & 1.036202E-08 & 6.384874E-02 \\
\hline 64 & $1.033428 \mathrm{E}-08$ & 6.389465E-02 \\
\hline 65 & $1.030655 \mathrm{E}-08$ & 6.394055E-02 \\
\hline 66 & $1.027881 \mathrm{E}-08$ & 6.398647E-02 \\
\hline 67 & 1.025107E-08 & $6.403238 \mathrm{E}-02$ \\
\hline 68 & 1.022334E-08 & $6.407828 \mathrm{E}-02$ \\
\hline 69 & $1.019560 \mathrm{E}-08$ & 6.412419E-02 \\
\hline 70 & $1.016786 \mathrm{E}-08$ & 6.417010E-02 \\
\hline 71 & 1.014013E-08 & 6.421601E-02 \\
\hline
\end{tabular}

Table 1. Fisssion yield data for $\mathrm{Ru}-101$ from each group fission 


\begin{tabular}{|l|l|l|}
\hline 72 & $1.011239 \mathrm{E}-08$ & $6.426191 \mathrm{E}-02$ \\
\hline 73 & $1.008465 \mathrm{E}-08$ & $6.430782 \mathrm{E}-02$ \\
\hline 74 & $1.005692 \mathrm{E}-08$ & $6.435373 \mathrm{E}-02$ \\
\hline
\end{tabular}

Table 2. Fission Yield Data for Important Nuclides

\begin{tabular}{|c|c|c|c|}
\hline No. & Nuclide & Independent & Cumulative \\
\hline 1 & 44101 & $1.39488412 \mathrm{E}-08$ & 0.0594559051 \\
\hline 2 & 46105 & $7.24691418 \mathrm{E}-11$ & 0.0528501496 \\
\hline 3 & 43099 & $8.94911238 \mathrm{E}-08$ & 0.0598406382 \\
\hline 4 & 45103 & $1.10945884 \mathrm{E}-10$ & 0.0682032108 \\
\hline 5 & 55133 & $1.2921422 \mathrm{E}-06$ & 0.0686191767 \\
\hline 6 & 46107 & $5.61074742 \mathrm{E}-08$ & 0.0328232199 \\
\hline 7 & 42097 & $8.35450635 \mathrm{E}-07$ & 0.0536595806 \\
\hline 8 & 62149 & $2.97196734 \mathrm{E}-09$ & 0.0122768953 \\
\hline 9 & 61147 & $1.76317592 \mathrm{E}-08$ & 0.0201525874 \\
\hline 10 & 60145 & $9.16564176 \mathrm{E}-08$ & 0.0293119382 \\
\hline 11 & 55135 & 0.000143690457 & 0 . \\
\hline 12 & 60143 & $5.13469302 \mathrm{E}-11$ & 0.0428884849 \\
\hline 13 & 54131 & $8.18919432 \mathrm{E}-07$ & 0.0390982628 \\
\hline 14 & 44102 & $4.00069894 \mathrm{E}-07$ & 0.0604525246 \\
\hline 15 & 62151. & $1.20191066 \mathrm{E}-06$ & 0.00770399207 \\
\hline 16 & 42095 & $4.79276396 \mathrm{E}-10$ & 0.0479308628 \\
\hline 17 & 42098. & $1.52272778 \mathrm{E}-05$ & 0.0569780767 \\
\hline 18 & 47109 & $1.54989905 \mathrm{E}-08$ & 0.0192497894 \\
\hline 19 & 44104 & $5.83728724 \mathrm{E}-05$ & 0.0599739552 \\
\hline 20 & 42100 & 0.00116378581 & 0.0667999163 \\
\hline 21 & 63153 & $1.04220319 \mathrm{E}-07$ & 0.00380806136 \\
\hline 22 & 40093 & $1.12774869 \mathrm{E}-05$ & 0.038283091 \\
\hline 23 & 44103 & $6.54340784 \mathrm{E}-06$ & 0.0682032108 \\
\hline 24 & 59141 & $6.37891129 \mathrm{E}-10$ & 0.0513297245 \\
\hline 25 & 53129 & $9.84646067 \mathrm{E}-06$ & 0.015332452 \\
\hline 26 & 40095 & 0.00111476786 & 0.0479257517 \\
\hline 27 & 40096 & 0.00546665257 & 0.049789656 \\
\hline 28 & 60146 & $2.0546679 \mathrm{E}-06$ & 0.0241217129 \\
\hline 29 & 54132 & $5.22244482 \mathrm{E}-05$ & 0.0537467673 \\
\hline 30 & 46108 & $1.02779777 \mathrm{E}-05$ & 0.0214385558 \\
\hline 31 & 41095 & $4.21908271 \mathrm{E}-06$ & 0.0479200929 \\
\hline 32 & 58141 & $4.06300796 \mathrm{E}-06$ & 0.0513297245 \\
\hline 33 & 40091 & $3.29098562 \mathrm{E}-08$ & 0.0246609803 \\
\hline 34 & 40092 & $2.57482975 \mathrm{E}-06$ & 0.0299894158 \\
\hline 35 & 54134 & 0.00104259967 & 0.0746262074 \\
\hline 36 & 44106 & 0.00478176028 & 0.0425091088 \\
\hline 37 & 62152 & $1.12275375 \mathrm{E}-05$ & 0.00586442789 \\
\hline 38 & 60148 & 0.000230132951 & 0.0161371008 \\
\hline 39 & 48111 & $9.53392365 \mathrm{E}-10$ & 0. \\
\hline 40 & 37085 & $3.76806099 \mathrm{E}-07$ & 0.00596903265 \\
\hline 41 & 53127 & $6.72185649 \mathrm{E}-08$ & 0. \\
\hline 42 & 57139 & $1.51741406 \mathrm{E}-05$ & 0. \\
\hline 43 & 46106 & $5.20088994 \mathrm{E}-09$ & 0.0425161459 \\
\hline 44 & 63155 & $6.24127051 \mathrm{E}-06$ & 0. \\
\hline 45 & 40094 & 0.000139707321 & 0.0433628 \\
\hline 46 & 62147 & $7.33138702 \mathrm{E}-13$ & 0.0201525874 \\
\hline 47 & 58142 & $5.83158871 \mathrm{E}-05$ & 0.0482551605 \\
\hline 48 & 60150 & 0.00257304939 & 0.00967459101 \\
\hline 49 & 60147 & $3.15005091 \mathrm{E}-05$ & 0.0201525111 \\
\hline 50 & 55137 & 0.00723981159 & 0.0654700324 \\
\hline 51 & 39091 & $3.37635788 \mathrm{E}-06$ & 0.024660904 \\
\hline 52 & 60144 & $2.12788609 \mathrm{E}-09$ & 0.0365167931 \\
\hline 53 & 36083 & $3.97525184 \mathrm{E}-07$ & 0.00309072481 \\
\hline 54 & 58144 & 0.00190069433 & 0.0365113653 \\
\hline 55 & 64157 & $5.38209179 \mathrm{E}-07$ & 0.000826512056 \\
\hline 56 & 46110 & 0.000235285333 & 0.00678964658 \\
\hline 57 & 42099 & 0.00016201062 & 0.0598405376 \\
\hline 58 & 64156 & $8.1606693 \mathrm{E}-08$ & 0.00128967955 \\
\hline 59 & 48113 & $8.94998422 \mathrm{E}-08$ & 0.00153323298 \\
\hline 60 & 55134 & $1.26644045 \mathrm{E}-05$ & $2.84286089 \mathrm{E}-05$ \\
\hline 61 & 63154 & $5.24785719 \mathrm{E}-07$ & $1.09989969 \mathrm{E}-06$ \\
\hline 62 & 58140 & $2.19996153 \mathrm{E}-07$ & 0.0540958382 \\
\hline 63 & 51125 & $9.95659284 \mathrm{E}-05$ & 0.00240752683 \\
\hline 64 & 65159 & $3.34624417 \mathrm{E}-08$ & 0.000259730499 \\
\hline 65 & 62154 & 0.000204086493 & 0.00275957375 \\
\hline 66 & 38090 & 0.000506891985 & 0.0211175736 \\
\hline 67 & 53131 & 0.00060731516 & 0.0390940532 \\
\hline 68 & 39089 & $6.96272018 \mathrm{E}-09$ & 0.0174264144 \\
\hline 69 & $\begin{array}{l}55003 \\
56138\end{array}$ & 0.00062428799 & $\begin{array}{l}0.01 / 4204144 \\
0.0590804704\end{array}$ \\
\hline 70 & 59143 & $4.51386285 \mathrm{E}-07$ & 0.0428884849 \\
\hline 71 & 35081 & $7.70846214 \mathrm{E}-07$ & 0. \\
\hline 72 & $\begin{array}{l}05001 \\
52130\end{array}$ & 0.00385167915 & 0.0249443837 \\
\hline
\end{tabular}

\begin{tabular}{|c|c|c|c|}
\hline 73 & 49115 & 4.8210044E-08 & 0.00102181779 \\
\hline 74 & 52128 & 0.000155515387 & 0.00909668952 \\
\hline 75 & 48112 & 7.40597983E-08 & 0.0022579045 \\
\hline 76 & 52129 & 0.000610249641 & 0.00200529373 \\
\hline 77 & 37087 & $6.0477927 \mathrm{E}-05$ & 0.0101353796 \\
\hline 78 & 36084 & 1.00259203E-05 & 0.00506843394 \\
\hline 79 & 54133 & 0.000174431727 & 0.0686179176 \\
\hline 80 & 51121 & $1.36808509 \mathrm{E}-07$ & 0.0013621588 \\
\hline 81 & 52127 & 2.26742177E-05 & 0.000875361147 \\
\hline 82 & 61148 & $2.81923462 \mathrm{E}-07$ & 2.81923462E-07 \\
\hline 83 & 34079 & $8.83708481 \mathrm{E}-07$ & 0.000518747373 \\
\hline 84 & 45105 & 5.52293386E-07 & 0.0528501496 \\
\hline 85 & 62150 & $8.83653115 \mathrm{E}-08$ & $2.21177816 \mathrm{E}-05$ \\
\hline 86 & 51123 & $1.0363422 \mathrm{E}-05$ & 0.00160690665 \\
\hline 87 & 64155 & $8.9122576 \mathrm{E}-09$ & 0.00179360155 \\
\hline 88 & 50117 & 1.08045128E-09 & 0.00131984882 \\
\hline 89 & 61149 & 5.79242806E-06 & 0.0122768888 \\
\hline 90 & 54136 & 0.0305194221 & 0.066190742 \\
\hline 91 & 46104 & $8.52468215 \mathrm{E}-13$ & $2.35028459 \mathrm{E}-08$ \\
\hline 92 & 64158 & $2.78086827 \mathrm{E}-06$ & 0.00048069976 \\
\hline 93 & 44100 & 3.88423099E-10 & $2.12777377 \mathrm{E}-06$ \\
\hline 94 & 36085 & $8.35864266 \mathrm{E}-05$ & 0.00133366848 \\
\hline 95 & 38089 & 5.34944957E-05 & 0.0174264014 \\
\hline 96 & 48114 & 3.77324818E-06 & 0.00144537922 \\
\hline 97 & 38088 & 4.13364023E-06 & 0.0140417218 \\
\hline 98 & 50119 & 1.50662146E-07 & 0.00122217531 \\
\hline 99 & 62148 & 6.33070263E-11 & 3.86537693E-07 \\
\hline 100 & 34082 & 0.000532393111 & 0.00213768682 \\
\hline 101 & 56136 & $2.21158416 \mathrm{E}-06$ & 0.00130924035 \\
\hline 102 & 47110 & $7.73662862 \mathrm{E}-07$ & $7.73662862 \mathrm{E}-07$ \\
\hline 103 & 34077 & 5.86704435E-11 & 9.47328372E-05 \\
\hline 104 & 36086 & 0.000686592888 & 0.00788113568 \\
\hline 105 & 63156 & $2.09438167 \mathrm{E}-05$ & 0.00128960051 \\
\hline 106 & 34080 & $1.54168374 \mathrm{E}-05$ & 0.0011528678 \\
\hline 107 & 63151 & $2.19690613 \mathrm{E}-10$ & 0.00770399207 \\
\hline 108 & 48116 & $8.04563824 \mathrm{E}-05$ & 0.00124024425 \\
\hline 109 & 50118 & 1.39797379E-07 & 0.00121764641 \\
\hline 110 & 48110 & 3.5894171E-10 & 9.93145591E-07 \\
\hline 111 & 34078 & 3.409949666E-08 & 0.000300992629 \\
\hline 112 & 54130 & $2.62221761 \mathrm{E}-07$ & 0.000116128431 \\
\hline 113 & 56137 & $2.25803033 \mathrm{E}-05$ & 0.0655588508 \\
\hline 114 & 64160 & $2.04950757 \mathrm{E}-05$ & 0.000141239143 \\
\hline 115 & 56140 & 0.010802621 & 0.0539481267 \\
\hline 116 & 50126 & 0.00207613781 & 0.0036603976 \\
\hline 117 & 52125 & $1.0644932 \mathrm{E}-07$ & 0.00240827538 \\
\hline 118 & 50120 & 6.40889766E-06 & 0.00119782588 \\
\hline
\end{tabular}

Table 3. the effect of reactor core dependent fision yield calculation

\begin{tabular}{|c|l|l|l|}
\hline $\begin{array}{l}\text { Burnup } \\
\text { Iteration } \\
\text { (100 days) }\end{array}$ & Original & Modif first it. & Modif second it. \\
\hline 1 & 1.157302 & 1.157302 & 1.157302 \\
\hline 2 & 1.157548 & 1.157578 & 1.157578 \\
\hline 3 & 1.158775 & 1.158837 & 1.158837 \\
\hline 4 & 1.159779 & 1.15987 & 1.159872 \\
\hline 5 & 1.160584 & 1.160707 & 1.160707 \\
\hline 6 & 1.161201 & 1.161357 & 1.161357 \\
\hline 7 & 1.161612 & 1.161797 & 1.161797 \\
\hline 8 & 1.161821 & 1.162039 & 1.162038 \\
\hline 9 & 1.161843 & 1.162092 & 1.162092 \\
\hline 10 & 1.161683 & 1.161962 & 1.161962 \\
\hline 11 & 1.161348 & 1.161657 & 1.161656 \\
\hline 12 & 1.160843 & 1.161183 & 1.161183 \\
\hline 13 & 1.16018 & 1.160551 & 1.160551 \\
\hline 14 & 1.159375 & 1.159774 & 1.159774 \\
\hline 15 & 1.158419 & 1.158845 & 1.158846 \\
\hline 16 & 1.157327 & 1.157782 & 1.157782 \\
\hline 17 & 1.156122 & 1.156607 & 1.156607 \\
\hline 18 & 1.154782 & 1.155295 & 1.155296 \\
\hline 19 & 1.153327 & 1.15387 & 1.15387 \\
\hline 20 & 1.151761 & 1.152329 & 1.152329 \\
\hline & & \multicolumn{2}{|l}{} \\
\hline
\end{tabular}

From the obtained data we found that to get highly accurate FP treatment in burn-up calculation we should modify the fission yield and burn-up chain 
in the cell burn-up calculation. We then insert these fission yield data to modify UCM66 burn-up-chain model in SRAC $^{9}$ and then we recalculate the cell calculation. The results are shown in table 3 . The first results(original) is just the calculation results using original UCM66 data. We then use the resulted spectrum to generate new fission yield distribution, modify the library and get modified first iteration result. The above process are repeated and the modified second iteration data.

From the above results we can see that the difference of Kinf calculation in Core 1 RBEC reactor after 2000 days is about $6 \times 10^{-2} \% \mathrm{dk} / \mathrm{k}$ or roughly $0.2 \$$ of reactivity for burnup level of $10 \% \mathrm{HM}$. His value is not significant in current conventional fast reactors, but for zero burnup core with higher burnup level the discrepancy may reach $0.5 \$$ and it clearly significant for nuclear reactor safety consideration especially at the end of life cycle. From the above results also we can see that the effect of further iteration is not important. The effect becomes more important in the nuclear reactors which adopt fuel recycling system ${ }^{13)}$.

\section{Conclusion}

The effect of fission yield calculation method on the nuclear fuel cell homogenization process has been investigated and discussed. This development is important to support implementation process of zero burn-up concept in inherently safe fast reactors. From the calculation results we can conclude that the difference of Kinf calculation in Core 1 RBEC reactor after 2000 days is about $6 \times 10^{-2} \% \mathrm{dk} / \mathrm{k}$ or roughly $0.2 \$$ of reactivity for burnup level of slightly less than\%HM. This value is not significant in current conventional fast reactors, but for zero burnup core with higher burnup level the discrepancy may reach $0.5 \$$ or more and it clearly significant for nuclear reactor safety consideration especially at the end of life cycle. The effect becomes more important in the nuclear reactors which adopt fuel recycling system.

\section{References}

1. H. Sekimoto and Zaki Su'ud, Design Study of Lead and Lead-bismuth Cooled Small Long-life Nuclear Power Reactors Using Metallic and Nitride Fuel, Nuclear Technology, 105:3, 1995.

2. Zaki Su'ud and H. Sekimoto, Safety Aspect of Long Life Small Safe Power Reactors, Annals of Nuclear Energy, 1995.
3. Zaki Su'ud and H. Sekimoto, Design and Safety Aspect of Lead and Lead Bismuth Cooled LongLife Small-safe Fast Reactor for Various Core Configuration, J. Nucl. Sci. Technol. 32/9, 1995.

4. Zaki Su'ud and H. Sekimoto, Accident Analysis of Lead or Lead-bismuth Cooled Small Safe Long-life Fast Reactor Using Metallic or Nitride Fuel, Nucl. Eng. Des. 162, 205-222, 1996.

5. Zaki Su'ud, Comparative Study on Safety Performance of Nitride Fueled Lead-Bismuth Cooled Fast Reactor with Various Power Level, Progress of Nuclear Energy, 32:3/4, pp. 571 577, 1998.

6. Zaki Su'ud, Neutronic Performance Comparation of MOX, Nitride and Metallic fuel Based 25-100 MWe Pb-Bi Cooled Long Life Fast Reactors without on site Refuelling, Progress of Nuclear Energy, 50, p. 276-278, 2008.

7. Zaki Su'ud, Safety Performance Comparation of MOX, Nitride and Metallic Fuel Based 25-100 MWe Pb-Bi Cooled Long Life Fast Reactors Without on Site Refuelling, Progress of Nuclear Energy, 50, p. 157-162, 2008.

8. D. Fitriani, et al., Design and Safety Optimization on Ship Based Small Nuclear Power Reactors, Progress of Nuclear Energy, 50,p. 299-303, 2008.

9. Zaki Su'ud et al., Performance of Benchmark Analysis for $\mathrm{Pb}-\mathrm{Bi} / \mathrm{Pb}$ Cooled Long-life Cores of Small Reactors Without On-Site Refuelling and Optimization of Their Inherent/passive Safety Performance, IAEA CRP Report, 2007

10. Zaki Su'ud et al., Performance of Benchmark Analysis for $\mathrm{Pb}-\mathrm{Bi} / \mathrm{Pb}$ Cooled Long-life Cores of Small Reactors Without On-Site Refuelling and Optimization of Their Inherent/passive Safety Performance, IAEA CRP Report, 2008.

11. K. Okumura et al., SRAC (Ver.2002); The Comprehensive Neutronics Calculation Code System, JAERI 2002.

12. S. Tabuchi and T. Aoyama, Lumped Group Constants of FP Nuclides for Fast Reactor Shielding Calculation Based on JENDL-3.2, wwn.dc.jaea.go.jp/nds/proceedings/2000/ p34.pdf

13. A. Waris et al., Influence of Void Fraction Change on Plutonium and Minor Actinides Recycling in BWR with Equilibrium Burnup, Journal of Progress in Nuclear Energy, 50, 2-6, 2008. 